\title{
Algebras of Reduced E-Fountain Semigroups and the Generalized Ample Identity
}

\author{
Itamar Stein* \\ Steinita@gmail.com
}

\begin{abstract}
Let $S$ be a reduced $E$-Fountain semigroup. If $S$ satisfies the congruence condition, there is a natural construction of a category $\mathcal{C}$ associated with $S$. We define a $\mathbb{k}$-module homomorphism $\varphi: \mathbb{k} S \rightarrow \mathbb{k} \mathcal{C}$ (where $\mathbb{k}$ is any unital commutative ring). With some assumptions, we prove that $\varphi$ is an isomorphism of $\mathbb{k}$-algebras if and only if some weak form of the right ample identity holds in $S$. This gives a unified generalization for a result of the author on right restriction E-Ehresmann semigroups and a result of Margolis and Steinberg on the Catalan monoid.
\end{abstract}

2020 Mathematics Subject Classification. 20M25, 16G10.

Keywords: Reduced E-Fountain semigroups, Semigroup algebras, Ample identity, Catalan monoid, Ehresmann semigroups.

\section{Introduction}

Let $S$ be a semigroup and let $\mathbb{k}$ be a commutative unital ring. It is of interest to study the semigroup algebra $\mathbb{k} S$ and its representations. Quite often it is complicated to investigate the algebra $\mathbb{k} S$ directly, but a change of basis yields an isomorphic algebra whose structure is more transparent. A pioneering work in this direction was done by Solomon [18] who proved that the semigroup algebra of a finite semilattice is isomorphic to a product of copies of the base ring. This was generalized by Steinberg [25] who showed that the algebra of any finite inverse semigroup is isomorphic to the algebra of its associated inductive groupoid. Steinberg's result is now fundamental in the study of representations of finite inverse semigroups. Guo and Chen [8] obtained a similar result for finite ample semigroups and the author extended this generalization to a class of right restriction

${ }^{*}$ Mathematics Unit, Shamoon College of Engineering, 77245 Ashdod, Israel 
E-Ehresmann semigroups [21, 22] (E-Ehresmann semigroups were introduced by Lawson in [12]). This result has led to several applications regarding semigroups of partial functions [20, 23, 24, 15] and recently also to the study of certain partition monoids [3. We mention also that Wang 28. generalized the above results further to a certain class of right $P$-restriction, $P$-Ehresmann semigroups (for definitions of these notions see [11]) - but we do not follow this approach in this paper. A hint for another direction is given by the Catalan monoid. The Catalan monoid $\mathrm{C}_{n}$ contains the order-preserving $(x \leq y \Longrightarrow f(x) \leq f(y))$ and order-increasing $(x \leq f(x))$ functions $f$ on $\{1, \ldots, n\}$. It is known that the algebra of the Catalan monoid is isomorphic to a certain incidence algebra ([9, Theorem 5.5] and [26, Theorem 17.25]) but recently Margolis and Steinberg obtained a simple proof of this fact using the change of basis approach [16]. Their result is not implied by any of the above-mentioned results. The goal of this paper is to obtain a generalization for the theorem on right restriction E-Ehresmann semigroups that includes also the case of the Catalan monoid. The class of semigroups which provides the correct context for this task is the class of reduced E-Fountain semigroups - also introduced by Lawson [13] under the name reduced $E$-semiabundant semigroups (which also appears in the literature as DR-semigroups [27]). Given a subset of idempotents $E$ of $S$ we can define two equivalence relations $\widetilde{\mathcal{L}}_{E}$ and $\widetilde{\mathcal{R}}_{E}$ on $S$. We say that $a \widetilde{\mathcal{L}}_{E} b\left(a \widetilde{\mathcal{R}}_{E} b\right)$ if $a$ and $b$ have the same set of right (respectively, left) identities from $E$. The semigroup $S$ is called reduced $E$-Fountain if every $\widetilde{\mathcal{L}}_{E}$ and $\widetilde{\mathcal{R}}_{E}$-class contains a (unique) idempotent from $E$ and $e f=e \Longleftrightarrow f e=e$ for every $e, f \in E$. If in addition $\widetilde{\mathcal{L}}_{E}$ and $\widetilde{\mathcal{R}}_{E}$ are right and left congruences respectively then we can associate a certain category $\mathcal{C}=\mathbf{C}(S)$ with the semigroup $S$ (for full details see [12]). We remark that $E$-Ehresmann semigroups are precisely those reduced $E$-Fountain semigroups which satisfy the congruence condition and whose distinguished subset of idempotents $E$ forms a subsemilattice (i.e., a commutative subsemigroup of idempotents). For $a, b \in S$ we define a relation $\unlhd_{l}$ by the rule that $a \unlhd_{l} b$ if $a=b e$ for an idempotent $e \in E$. This is a generalization of the right restriction partial order defined on $E$-Ehresmann semigroups - but $\unlhd_{l}$ is not a partial order or even a preorder. We also define an identity we call the generalized right ample condition which is a weak form of the right ample condition studied in the theory of E-Fountain semigroups. This background is described in Section 2 and Section 3 of the paper. Let $S$ be a reduced $E$-Fountain semigroup which satisfies the congruence condition and assume also that sets of the form $\left\{b \in S \mid b \unlhd_{l} a\right\}$ are finite for every $a \in S$. Let $\mathbb{k}$ be a commutative unital ring. In Section 4 we define a "change of basis" $\mathbb{k}$-module homomorphism $\varphi$ between the semigroup algebra $\mathbb{k} S$ and $\mathbb{k} \mathcal{C}$ - the algebra of the associated category $\mathcal{C}$. We show that $\varphi$ is a homomorphism of $\mathbb{k}$-algebras if and only if the generalized right ample identity holds. We also obtain an isomorphism in case $\unlhd_{l}$ is contained in a partial order. In Section 5 we consider the case where $E$ is a subband (i.e., a subsemigroup of idempotents). In this case, we obtain an $E$-Ehresmann semigroup and $\unlhd_{l}$ is a partial order. Moreover, the generalized right ample condition and the standard one coincide. In Section 6 we discuss in detail the case of the Catalan monoid and show that we retrieve the 
isomorphism described in [16].

Acknowledgments: The author thanks Professor Victoria Gould for a helpful conversation and the referee for helpful comments.

\section{Preliminaries}

Let $S$ be a semigroup and let $S^{1}=S \cup\{1\}$ be the monoid formed by adjoining a formal unit element. Recall that Green's preorders $\leq_{\mathcal{R}}, \leq_{\mathcal{L}}$ and $\leq_{\mathcal{J}}$ are defined by:

$$
\begin{aligned}
& a \leq_{\mathcal{R}} b \Longleftrightarrow a S^{1} \subseteq b S^{1} \\
& a \leq_{\mathcal{L}} b \Longleftrightarrow S^{1} a \subseteq S^{1} b \\
& a \leq_{\mathcal{J}} b \Longleftrightarrow S^{1} a S^{1} \subseteq S^{1} b S^{1}
\end{aligned}
$$

The associated Green's equivalence relations on $S$ are denoted by $\mathcal{R}, \mathcal{L}$ and $\mathcal{J}$. It is well known that $\mathcal{L}(\mathcal{R})$ is a right congruence (respectively, left congruence). A semigroup $S$ is called $\mathcal{J}$ - trivial if $\mathcal{J}$ is the identity relation (that is, $a \mathcal{J} b \Longleftrightarrow a=b$ ). Similar definitions hold for $\mathcal{R}$-trivial and $\mathcal{L}$-trivial semigroups. We denote by $E(S)$ the set of idempotents of $S$. We denote by $\leq$ the natural partial order on $E(S)$ defined by

$$
e \leq f \Longleftrightarrow(e f=f e=e)
$$

Other elementary semigroup theoretic notions can be found in [10]. Let $E \subseteq E(S)$ be some subset of idempotents. For every $a \in S$ we denote by $a_{E}$ the set of right identities of $a$ from $E$ :

$$
a_{E}=\{e \in E \mid a e=a\}
$$

Dually $_{E} a$ is the set of left identities from $E$. We define two equivalence relations $\widetilde{\mathcal{L}}_{E}$ and $\widetilde{\mathcal{R}}_{E}$ on $S$ by

$$
\begin{aligned}
a \widetilde{\mathcal{L}}_{E} b & \Longleftrightarrow\left(a_{E}=b_{E}\right) \\
a \widetilde{\mathcal{R}}_{E} b & \Longleftrightarrow\left({ }_{E} a={ }_{E} b\right) .
\end{aligned}
$$

It is easy to see that $\mathcal{L} \subseteq \widetilde{\mathcal{L}}_{E}$ and $\mathcal{R} \subseteq \widetilde{\mathcal{R}}_{E}$. Recall that a subsemigroup of idempotents $E \subseteq S$ is called a subband. A commutative subband is called a subsemilattice.

Let $\mathbb{k}$ be a commutative unital ring. The semigroup algebra $\mathbb{k} S$ of a semigroup $S$ is defined in the following way. It is a free $\mathbb{k}$-module with basis the elements of $S$, that is, it consists of all formal 
linear combinations

$$
\left\{k_{1} s_{1}+\ldots+k_{n} s_{n} \mid k_{i} \in \mathbb{k}, s_{i} \in S\right\} .
$$

The multiplication in $\mathbb{k} S$ is the linear extension of the semigroup multiplication. We will also need the notion of a category algebra in this paper. The category algebra $\mathbb{k} \mathcal{C}$ of a (small) category $\mathcal{C}$ is defined in the following way. It is a free $\mathbb{k}$-module with the morphisms of $\mathcal{C}$ as a basis, that is, it consists of all formal linear combinations

$$
\left\{k_{1} m_{1}+\ldots+k_{n} m_{n} \mid k_{i} \in \mathbb{k}, m_{i} \in \mathcal{C}^{1}\right\}
$$

The multiplication in $\mathbb{k} \mathcal{C}$ is the linear extension of the following:

$$
m^{\prime} \cdot m= \begin{cases}m^{\prime} m & \text { if } m^{\prime} m \text { is defined } \\ 0 & \text { otherwise }\end{cases}
$$

Let $R$ be a relation on a set $X$. We say that $R$ is principally finite if the set $\left\{x^{\prime} \in X \mid x^{\prime} R x\right\}$ is finite for every $x \in X$. If $R=\preceq$ is a principally finite partial order, we can define the incidence algebra of $\preceq$ which consists of all functions $f: \preceq \rightarrow \mathbb{k}$ with standard addition operation and whose multiplication operation is defined by

$$
f \star g(a, b)=\sum_{a \preceq c \preceq b} f(a, c) g(c, b) .
$$

We denote this algebra by $\mathbb{k}[\preceq]$. It is well known that an element $f \in \mathbb{k}[\preceq]$ is invertible if and only if $f(x, x)$ is invertible in $\mathbb{k}$ for every $x \in X$. For other elementary facts on incidence algebras see [19, Section 3.6]). Another point is worth mentioning. The poset $\preceq$ can also be viewed as a category whose set of objects is $X$ and there exists a unique morphism from an object $x_{1}$ to another object $x_{2}$ if and only if $x_{1} \preceq x_{2}$. The category algebra in general differs from the incidence algebra because the elements of the category algebra are only finite linear combinations. However, the two notions coincide if $X$ is a finite set.

\section{Reduced E-Fountain Semigroups}

\subsection{Basic definitions}

Definition 3.1. A semigroup $S$ is called E-Fountain if every $\widetilde{\mathcal{L}}_{E}$-class contains an idempotent from $E$ and every $\widetilde{\mathcal{R}}_{E}$-class contains an idempotent from $E$, where $E \subseteq S$ is some subset of idempotents. We remark that this property is also called " $E$-semiabundant" in the literature. 
The following is an immediate consequence of [27, Propositions 1.2 and 1.3].

Proposition 3.2. Let $S$ be an E-Fountain semigroup. The following conditions are equivalent:

1. $\forall e, f \in E, \quad$ ef $=e \Longleftrightarrow f e=e$.

2. For every $a \in S$ the sets $a_{E}$ and ${ }_{E}$ a contain a minimum element (with respect to the natural partial order on idempotents).

3. We can equip $S$ with two unary operations $*$ and + which satisfy the following identities for every $a, b \in S$ :

$$
\begin{aligned}
& a^{+} a=a \quad\left(a^{+}\right)^{+}=a^{+} \quad a^{+}(a b)^{+}=(a b)^{+} a^{+}=(a b)^{+} \\
& a a^{*}=a \quad\left(a^{*}\right)^{*}=a^{*} \quad b^{*}(a b)^{*}=(a b)^{*} b^{*}=(a b)^{*} \\
& \left(a^{+}\right)^{*}=a^{+}\left(a^{*}\right)^{+}=a^{*}
\end{aligned}
$$

It is important to note that $a^{*}\left(a^{+}\right)$is the minimum element of $a_{E}$ (respectively, ${ }_{E} a$ ) and that $e^{*}=e^{+}=e$ for every $e \in E$.

We follow [13] and call an E-Fountain semigroup reduced if it satisfies the equivalent conditions of Proposition 3.2. Such a semigroup is called a "DR-semigroup" in [27. It is clear from Condition 3 that the class of all reduced $E$-Fountain semigroup is a variety of bi-unary semigroups.

Definition 3.3. Let $S$ be a reduced $E$-Fountain semigroup. We say that $S$ satisfies the congruence condition if $\widetilde{\mathcal{L}}_{E}$ is a right congruence and $\widetilde{\mathcal{R}}_{E}$ is a left congruence.

It is well known that $S$ satisfies the congruence condition if and only if the identities $(a b)^{*}=\left(a^{*} b\right)^{*}$ and $(a b)^{+}=\left(a b^{+}\right)^{+}$hold - see [6, Lemma 4.1]. In this case we can define a category $\mathcal{C}(S)$ in the following way. The objects are in one-to-one correspondence with the set $E$. The morphisms are in one to one correspondence with elements of $S$. The convention is to compose morphisms in such categories "from left to right". However, for us it will be more convenient to use composition "from right to left". Therefore, for every $a \in S$ the associated morphism $C(a)$ has domain $a^{*}$ and range $a^{+}$. If the range of $C(a)$ is the domain of $C(b)$ (that is, if $b^{*}=a^{+}$) the composition $C(b) \cdot C(a)$ is defined to be $C(b a)$. The assumption $b^{*}=a^{+}$implies that $(b a)^{+}=\left(b a^{+}\right)^{+}=\left(b b^{*}\right)^{+}=b^{+}$and likewise $(b a)^{*}=a^{*}$ so this is indeed a category - see [12] for additional details.

\subsection{The relations $\leq_{l}$ and $\unlhd_{l}$}

Let $S$ be a reduced $E$-Fountain semigroup. The following partial order is defined in [12:

$$
a \leq_{l} b \Longleftrightarrow\left(a^{*} \leq b^{*} \text { and } a=b a^{*}\right)
$$


This is a generalization of the right "restriction" partial order of an E-Ehresmann semigroup and in particular of the natural partial order of an inverse semigroup. However, we will need a different generalization for our purpose.

Definition 3.4. Let $a, b \in S$. We define $a \unlhd_{l} b \Longleftrightarrow a=b e$ for some $e \in E$.

Lemma 3.5. $a \unlhd_{l} b \Longleftrightarrow a=b a^{*}$.

Proof. The $\Leftarrow$ direction is immediate. For the other direction assume $a=b e$ so $a e=a$ hence $a^{*} \leq e$ in $E$. Therefore

$$
a=a a^{*}=a\left(e a^{*}\right)=(a e) a^{*}=b a^{*}
$$

as required.

The following uniqueness property will also be useful.

Lemma 3.6. If $a \unlhd_{l} b$ then $a$ is the unique $x \in S$ such that $x \unlhd_{l} b$ and $x^{*}=a^{*}$.

Proof. Assume $x \unlhd_{l} b$ such that $x^{*}=a^{*}$. Then $x=b x^{*}=b a^{*}=a$.

It is clear that $\leq_{l} \subseteq \unlhd_{l}$ and it is easy to see that $e \leq f \Longleftrightarrow e \leq_{l} f \Longleftrightarrow e \unlhd_{l} f$ for $e, f \in E$. It is tempting to think that $\unlhd_{l}$ is also a partial order. It is clear that $\unlhd_{l}$ is reflexive since $a=a a^{*}$. However, the following example shows that in general it is not antisymmetric and in Remark 6.9 we will give a counter-example for transitivity.

Example 3.7. [28, Example 2.3] Let $S$ be a "square" rectangular band. The elements of $S$ are pairs $(i, j)$ where $1 \leq i, j \leq n$ for some fixed $n \in \mathbb{N}$. Multiplication is defined by

$$
\left(i_{1}, j_{1}\right) \cdot\left(i_{2}, j_{2}\right)=\left(i_{1}, j_{2}\right)
$$

Recall that $\left(i_{1}, j_{1}\right) \mathcal{R}\left(i_{2}, j_{2}\right) \Longleftrightarrow i_{1}=i_{2}$ and $\left(i_{1}, j_{1}\right) \mathcal{L}\left(i_{2}, j_{2}\right) \Longleftrightarrow j_{1}=j_{2}$. Every element of $S$ is an idempotent, but we choose $E$ to be the set of "diagonal" elements $E=\{(i, i) \mid 1 \leq i \leq n\}$. It is easy to see that

$$
(i, j) e=(i, j) \Longleftrightarrow e=(j, j)
$$

for $e \in E$. Therefore, the set of right identities of $(i, j)$ contains a unique idempotent from $E$ and the dual claim holds for left identities. Hence, $S$ is a reduced $E$-Fountain semigroup. It is also easy to check that $\left(i_{1}, j_{1}\right) \unlhd_{l}\left(i_{2}, j_{2}\right)$ if and only if $i_{1}=i_{2}$. Therefore, $a \unlhd_{l} b$ if and only if $a \mathcal{R} b$ so $\unlhd_{l}$ is in fact a symmetric relation! On the other hand, $\leq$ is the trivial relation on $E$ so $\leq_{l}$ is also trivial on $S$. We give another simple observation for future use. It is easy to verify that $\left(i_{1}, j_{1}\right) \widetilde{\mathcal{L}}_{E}\left(i_{2}, j_{2}\right)$ if and only if $j_{1}=j_{2}$. Therefore, $\widetilde{\mathcal{L}}_{E}=\mathcal{L}$ so $\widetilde{\mathcal{L}}_{E}$ is a right congruence and and dually $\widetilde{\mathcal{R}}_{E}=\mathcal{R}$ is a left congruence so $S$ satisfies also the congruence condition. 
Clearly, we can also define a dual relation $a \unlhd_{r} b \Longleftrightarrow a=e b$ for some $e \in E$.

\subsection{The right ample and the generalized right ample conditions}

Let $S$ be a reduced $E$-Fountain semigroup which satisfies the congruence condition. We say that the right ample condition (or right ample identity) holds in $S$ if $e a=a(e a)^{*}$ for every $a \in S$ and $e \in E$. This is equivalent to the condition $E a \subseteq a E$ for every $a \in S$. The right ample condition is well studied, but it is a too strong requirement for some of the monoids we want to consider as we immediately show.

Lemma 3.8. If the right ample condition holds then $E$ is a subband of $S$.

Proof. Let $e, f \in E$. First note that $(e f)^{*} \leq f$ since $e f f=e f$. Now, the right ample identity implies that

$$
e f=f(e f)^{*}=(e f)^{*} \in E
$$

so $E$ is a subband.

Lemma 3.9. Let $S$ be a reduced E-Fountain semigroup. If $E$ is a subband of $S$ then $E$ is a subsemilattice.

Proof. Let $e, f \in E$. The fact that $e(e f)=e f$ implies that $(e f) e=e f$. Likewise, $(f e) e=f e$ implies efe $=f e$ so $e f=f e$.

Definition 3.10. A reduced $E$-Fountain semigroup which satisfies the congruence condition is called E-Ehresmann [6, 7] if $E$ is a subsemilattice of $S$.

If we consider reduced E-Fountain semigroups, Lemma 3.8 and Lemma 3.9 show that the right ample condition is relevant only in the context of E-Ehresmann semigroups. We want a condition which is applicable also for reduced $E$-Fountain semigroups where $E$ is not a subband.

Definition 3.11. Let $S$ be a reduced $E$-Fountain semigroup which satisfies the congruence condition. We say that the generalized right ample condition (or generalized right ample identity) holds in $S$ if

$$
\left(e\left(a(e a f)^{*}\right)^{+}\right)^{*}=\left(a(e a f)^{*}\right)^{+}
$$

for every $a \in S$ and $e, f \in E$.

This identity can also be written as

$$
\left(b^{*}\left(a\left(b^{*} a c^{*}\right)^{*}\right)^{+}\right)^{*}=\left(a\left(b^{*} a c^{*}\right)^{*}\right)^{+}
$$


for every $a, b, c \in S$ hence the class of reduced $E$-Fountain semigroups which satisfy the congruence condition and the generalized right ample identity is also a variety of bi-unary semigroups.

Next, we show that if $S$ satisfies the right ample condition it satisfies also the generalized right ample condition hence justifying our term "generalized". We fix a reduced E-Fountain semigroup $S$ which satisfies the congruence condition.

Lemma 3.12. If $E$ is a subsemilattice then $a \unlhd_{l} b \Longrightarrow a^{*} \leq b^{*}$.

Proof. If $a=b a^{*}$ then $a b^{*}=b a^{*} b^{*}=b b^{*} a^{*}=b a^{*}=a$ so $a^{*} \leq b^{*}$.

We record the following immediate corollary.

Corollary 3.13. If $E$ is a subsemilattice then $\unlhd_{l}=\leq_{l}$.

Proposition 3.14. If the right ample condition holds in $S$ then so does the generalized right ample condition.

Proof. Let $e, f \in E$ and $a \in S$. By Lemma 3.8 and Lemma 3.9 we know that $E$ is a semilattice. Since eaf $\unlhd_{l}$ ea, Lemma 3.12 implies that $(e a)^{*}(e a f)^{*}=(e a f)^{*}$. This and the right ample identity implies that

$$
e a(e a f)^{*}=a(e a)^{*}(e a f)^{*}=a(e a f)^{*}
$$

so $e$ is a left identity of $a(e a f)^{*}$. Since $a(\text { eaf })^{*} \widetilde{\mathcal{R}}_{E}\left(a(e a f)^{*}\right)^{+}$we obtain $e\left(a(e a f)^{*}\right)^{+}=\left(a(e a f)^{*}\right)^{+}$ as well. Finally,

$$
\left(e\left(a(e a f)^{*}\right)^{+}\right)^{*}=\left(\left(a(e a f)^{*}\right)^{+}\right)^{*}=\left(a(e a f)^{*}\right)^{+}
$$

so $S$ satisfies the generalized right ample condition.

Remark 3.15. We can of course define the dual notion. We say that $S$ satisfies the generalized left ample condition if the identity

$$
\left(\left((f a e)^{+} a\right)^{*} e\right)^{+}=\left((f a e)^{+} a\right)^{*}
$$

holds for every $a \in S$ and $e, f \in E$.

We give here two examples of semigroups which satisfy the generalized right ample condition but not the standard one. Although the main theorem of this paper (Theorem 4.4) is not applicable in these examples as we will see later, we hope they offer conviction that the generalized right ample identity is not an artificial condition. The main motivating example - the Catalan monoid - is postponed until Section 6 . 
Example 3.16. The rectangular band from Example 3.7 satisfies the generalized right ample condition but not the right ample condition. If $\left(a(e a f)^{*}\right)^{+}=(i, i)$ and $e=(k, k)$ then

$$
\left(e\left(a(e a f)^{*}\right)^{+}\right)^{*}=((k, k)(i, i))^{*}=(k, i)^{*}=(i, i)=\left(a(e a f)^{*}\right)^{+}
$$

so the required equality holds for every $e, f \in E$, and $a \in S$. On the other hand, $E$ is not a band so the right ample condition doesn't hold. We remark that it is equally easy to see that the left generalized ample identity holds in this case.

Example 3.17 ([27). Let $H$ be a Hilbert space and let $L(H)$ be the algebra of all bounded linear operators on $H$ (this is one of the main examples of a Rickart $*$-ring and a Baer $*$-ring [1]). For every closed subspace $U \subseteq H$ we associate the orthogonal projection $P_{U} \in L(H)$ onto it. Recall that $P_{U}$ is an idempotent and $\operatorname{im}\left(P_{U}\right)=U=\operatorname{ker}\left(P_{U}\right)^{\perp}($ where $\operatorname{im} T$ and $\operatorname{ker} T$ are the image and kernel of the linear operator $T$ and $V^{\perp}$ is the orthogonal complement of $V$ ). It is easy to check that

$$
P_{U} P_{V}=P_{U} \Longleftrightarrow P_{V} P_{U}=P_{U} \Longleftrightarrow U \subseteq V
$$

Now, consider $L(H)$ as a multiplicative monoid. This is an example of a Baer $*$-semigroup [4]. If we set $E=\left\{P_{U} \mid U\right.$ is a closed subspace of $\left.H\right\}$, it is not difficult to check that for $T, S \in L(H)$

$$
T \widetilde{\mathcal{L}}_{E} S \Longleftrightarrow \operatorname{ker}(T)=\operatorname{ker}(S), \quad T \widetilde{\mathcal{R}}_{E} S \Longleftrightarrow \operatorname{im}(T)=\operatorname{im}(S)
$$

It follows that $L(H)$ is a reduced $E$-Fountain semigroup where $T^{*}=P_{(\operatorname{ker} T)^{\perp}}$ and $T^{+}=P_{\mathrm{im}(T)}$.

Lemma 3.18. $L(H)$ satisfies the congruence condition.

Proof. Let $T, S, R \in L(H)$. First assume $T \widetilde{\mathcal{L}}_{E} S$ so $\operatorname{ker}(T)=\operatorname{ker}(S)$. We have

$$
x \in \operatorname{ker}(T R) \Longleftrightarrow R(x) \in \operatorname{ker} T \Longleftrightarrow R(x) \in \operatorname{ker} S \Longleftrightarrow x \in \operatorname{ker}(S R)
$$

so $\operatorname{ker}(T R)=\operatorname{ker}(S R)$ and $\widetilde{\mathcal{L}}_{E}$ is a right congruence. If $T \widetilde{\mathcal{R}}_{E} S$ then $\operatorname{im}(T)=\operatorname{im}(S)$. In this case

$$
x \in \operatorname{im}(R T) \Longleftrightarrow \exists y \in \operatorname{im}(T), \quad R(y)=x \Longleftrightarrow \exists y \in \operatorname{im}(S), \quad R(y)=x \Longleftrightarrow x \in \operatorname{im}(R S)
$$

so $\widetilde{\mathcal{R}}_{E}$ is a left congruence.

Note that in general $P_{U} P_{V} \neq P_{V} P_{U}$ so the right ample identity does not hold and $L(H)$ is not an E-Ehresmann semigroup.

Lemma 3.19. $L(H)$ satisfies the generalized right ample identity. 
Proof. Let $a \in L(H)$ and $e, f \in E$. Set $e=P_{U}$, (eaf $)^{*}=P_{W}$ and $\left(a(e a f)^{*}\right)^{+}=P_{V}$. We need to show that $\left(P_{U} P_{V}\right)^{*}=P_{V}$, which means that $\operatorname{ker}\left(P_{U} P_{V}\right)=\operatorname{ker}\left(P_{V}\right)$. This is equivalent to $\operatorname{im}\left(P_{V}\right) \cap \operatorname{ker}\left(P_{U}\right)=0$ so we need to prove that $V \cap U^{\perp}=0$ where $V=\operatorname{im}\left(a\left(P_{U} a f\right)^{*}\right)$. Let $y \in V \cap U^{\perp}$, then $y=a(x)$ for $x \in \operatorname{im}\left(P_{U} a f\right)^{*}=W=\operatorname{ker}\left(P_{U} a f\right)^{\perp}$. The fact that $\operatorname{ker}(f) \subseteq$ $\operatorname{ker}\left(P_{U} a f\right)$ implies that $W=\operatorname{ker}\left(P_{U} a f\right)^{\perp} \subseteq \operatorname{ker}(f)^{\perp}$. Since $f \in E, \operatorname{ker}(f)^{\perp}=\operatorname{im}(f)$ so $f(x)=x$ and

$$
P_{U} a f(x)=P_{U} a(x)=P_{U}(y)=0
$$

since $y \in U^{\perp}$. This shows that $x \in \operatorname{ker}\left(P_{U} a f\right)$, but we already know that $x \in \operatorname{ker}\left(P_{U} a f\right)^{\perp}$ so $x=0$ and $y=a(x)=0$ as required.

Remark 3.20. $L(H)$ satisfies also the left ample identity. For $T \in L(H)$ we denote by $T^{t}$ its adjoint operator (we do not use $T^{*}$ here to avoid confusion with the unary operation). It is clear that $\left(P_{U}\right)^{t}=P_{U},\left(T^{t}\right)^{+}=T^{*}$ and $\left(T^{t}\right)^{*}=T^{+}$so every identity immediately implies its dual in $L(H)$.

This example will not be considered further in this paper because it does not satisfy the finiteness condition we will require later.

\section{The semigroup and category algebras}

\subsection{Homomorphism of algebras}

In this section we fix a reduced $E$-Fountain semigroup $S$ which satisfies the congruence condition. We also assume that the relation $\unlhd_{l}$ is principally finite. Let $\mathcal{C}$ be the associated category as defined in Section 3 and let $\mathbb{k}$ be a commutative unital ring. Define $\varphi: \mathbb{k} S \rightarrow \mathbb{k} \mathcal{C}$ on basis elements by

$$
\varphi(a)=\sum_{c \unlhd_{l} a} C(c) .
$$

It is clear that $\varphi$ is a $\mathbb{k}$-module homomorphism. We want to show that it is an algebra homomorphism if and only if the generalized right ample condition holds in $S$. For this we need a different formulation of this identity.

Lemma 4.1. The following conditions are equivalent.

1. The semigroup $S$ satisfies the generalized right ample condition.

2. The implication $c \unlhd_{l}$ ea $\Longrightarrow\left(e\left(a c^{*}\right)^{+}\right)^{*}=\left(a c^{*}\right)^{+}$holds for every $a, c \in S$ and $e \in E$.

3. The implication $c \unlhd_{l} b a \Longrightarrow\left(b\left(a c^{*}\right)^{+}\right)^{*}=\left(a c^{*}\right)^{+}$holds for every $a, b, c \in S$. 
Proof.

$(1 \Longrightarrow 2)$ If $c \unlhd_{l}$ ea then $c=e a f$ for some $f \in E$. The equality $\left(e\left(a c^{*}\right)^{+}\right)^{*}=\left(a c^{*}\right)^{+}$follows immediately from the generalized right ample identity.

$(2 \Longrightarrow 3)$ Assume $c \unlhd_{l} b a$ so $c=b a c^{*}$. Denote $d=b^{*} a c^{*}$ so clearly $d \unlhd_{l} b^{*} a$. Our assumption implies

$$
\left(b^{*}\left(a d^{*}\right)^{+}\right)^{*}=\left(a d^{*}\right)^{+} .
$$

Now,

$$
d^{*}=\left(b^{*} a c^{*}\right)^{*}=\left(b a c^{*}\right)^{*}=c^{*}
$$

so the above equality can be written

$$
\left(b^{*}\left(a c^{*}\right)^{+}\right)^{*}=\left(a c^{*}\right)^{+} .
$$

Therefore,

$$
\left(b\left(a c^{*}\right)^{+}\right)^{*}=\left(b^{*}\left(a c^{*}\right)^{+}\right)^{*}=\left(a c^{*}\right)^{+}
$$

which finishes the proof.

$(3 \Longrightarrow 1)$ First substitute $b=e$ for $e \in E$ and then note that $e a f \unlhd_{l}$ ea so we can choose $c=e a f$ and the assumption implies

$$
\left(e\left(a(e a f)^{*}\right)^{+}\right)^{*}=\left(a(e a f)^{*}\right)^{+}
$$

as required.

Theorem 4.2. The module homomorphism $\varphi$ is a homomorphism of $\mathbb{k}$-algebras if and only if the generalized right ample identity holds in $S$.

Proof. We start with the "if" part. For $a, b \in S$, we need to show that $\varphi(b a)=\varphi(b) \varphi(a)$. We need to prove that

$$
\sum_{c \unlhd_{l} b a} C(c)=\sum_{c^{\prime \prime} \unlhd_{l} b} C\left(c^{\prime \prime}\right) \sum_{c^{\prime} \unlhd_{l} a} C\left(c^{\prime}\right) .
$$

First note that if $c^{\prime \prime} \unlhd_{l} b$ and $c^{\prime} \unlhd_{l} a$ and $C\left(c^{\prime \prime}\right) C\left(c^{\prime}\right) \neq 0$ then $\left(c^{\prime \prime}\right)^{*}=\left(c^{\prime}\right)^{+}$and so

$$
c^{\prime \prime} c^{\prime}=b\left(c^{\prime \prime}\right)^{*} c^{\prime}=b\left(c^{\prime}\right)^{+} c^{\prime}=b c^{\prime}=b a\left(c^{\prime}\right)^{*}
$$

so $c^{\prime \prime} c^{\prime} \unlhd_{l} b a$. Therefore, every element on the right-hand side appears also on the left-hand side. Now, take $c \unlhd_{l} b a$ and define $c^{\prime}=a c^{*}$ and $c^{\prime \prime}=b\left(a c^{*}\right)^{+}$. From the generalized right ample condition 
we obtain

$$
\left(c^{\prime \prime}\right)^{*}=\left(b\left(a c^{*}\right)^{+}\right)^{*}=\left(a c^{*}\right)^{+}=\left(c^{\prime}\right)^{+} .
$$

Therefore, the composition $C\left(c^{\prime \prime}\right) \cdot C\left(c^{\prime}\right)$ is defined. Now

$$
c^{\prime \prime} c^{\prime}=b\left(a c^{*}\right)^{+} a c^{*}=b a c^{*}=c
$$

so every element from the left-hand side appears on the right-hand side. It remains to show that it appears only once. Assume $C(c)=C\left(d^{\prime \prime}\right) C\left(d^{\prime}\right)$ for $d^{\prime \prime} \unlhd_{l} b$ and $d^{\prime} \unlhd_{l} a$. Then $\left(d^{\prime}\right)^{*}=c^{*}$ so by Lemma 3.6 we must have $d^{\prime}=a c^{*}=c^{\prime}$. Since $C\left(d^{\prime \prime}\right) \cdot C\left(d^{\prime}\right)$ is defined we must have $\left(d^{\prime \prime}\right)^{*}=\left(d^{\prime}\right)^{+}=\left(a c^{*}\right)^{+}$so again Lemma 3.6 implies $d^{\prime \prime}=b\left(a c^{*}\right)^{+}=c^{\prime \prime}$. This proves uniqueness.

The "only if" part comes from another examination of the above argument. Let $a, b \in S$, if $\varphi$ is a homomorphism of algebras we must have $\varphi(b a)=\varphi(b) \varphi(a)$ so

$$
\sum_{c \unlhd_{l} b a} C(c)=\sum_{c^{\prime \prime} \unlhd_{l} b} C\left(c^{\prime \prime}\right) \sum_{c^{\prime} \unlhd_{l} a} C\left(c^{\prime}\right)
$$

Choose any $c \unlhd_{l} b a$ from the left-hand side. The morphism $C(c)$ must appear on the right-hand side. So there exists $c^{\prime} \unlhd_{l} a$ and $c^{\prime \prime} \unlhd_{l} b$ such that $C(c)=C\left(c^{\prime \prime}\right) C\left(c^{\prime}\right)$. This implies $\left(c^{\prime}\right)^{*}=c^{*}$ so $c^{\prime}=a c^{*}$. Since the product $C(c)=C\left(c^{\prime \prime}\right) C\left(c^{\prime}\right)$ is defined it must be the case that $\left(c^{\prime \prime}\right)^{*}=\left(a c^{*}\right)^{+}$ and therefore $c^{\prime \prime}=b\left(a c^{*}\right)^{+}$. This implies $\left(b\left(a c^{*}\right)^{+}\right)^{*}=\left(a c^{*}\right)^{+}$as required.

\subsection{Isomorphism of algebras}

In general, $\varphi$ is not an isomorphism. For instance, consider Example 3.7 We have already seen (Example 3.16) that it satisfies the requirements of Theorem 4.2 so $\varphi$ is an homomorphism of $\mathbb{k}$-algebras. But in this example we have $\varphi(a)=\varphi(b)$ if $a \mathcal{R} b$ so $\varphi$ is not injective.

However, we can prove that $\varphi$ is an isomorphism if $\unlhd_{l}$ is contained in a (principally finite) partial order.

Lemma 4.3. Let $S$ be a reduced E-Fountain semigroup which satisfies the congruence condition. Assume $\unlhd_{l} \subseteq \preceq$ where $\preceq$ is some principally finite partial order. Then $\varphi$ is an isomorphism of $\mathbb{k}$-modules.

Proof. Consider the incidence algebra $\mathbb{k}[\preceq]$. Define $\zeta_{l} \in \mathbb{k}[\preceq]$ to be the zeta function of $\unlhd_{l}$ :

$$
\zeta_{l}(a, b)= \begin{cases}1 & a \unlhd_{l} b \\ 0 & \text { otherwise }\end{cases}
$$


As $\zeta_{l}(a, a)=1$ for every $a \in A$, we know that $\zeta_{l}$ has an inverse $\zeta_{l}^{-1}$. This means that

$$
\zeta_{l}^{-1} \star \zeta_{l}(a, b)=\sum_{a \preceq c \preceq b} \zeta_{l}^{-1}(a, c) \zeta_{l}(c, b)=\delta(a, b)= \begin{cases}1 & a=b \\ 0 & a \neq b\end{cases}
$$

and likewise

$$
\zeta_{l} \star \zeta_{l}^{-1}(a, b)=\delta(a, b)
$$

Note that

$$
\varphi(a)=\sum_{b \unlhd_{l} a} C(b)=\sum_{b \preceq a} \zeta_{l}(b, a) C(b) .
$$

The inverse of $\varphi$ is given by

$$
\psi(C(a))=\sum_{b \preceq a} \zeta_{l}^{-1}(b, a) b .
$$

Indeed

$$
\begin{aligned}
\psi(\varphi(a)) & =\psi\left(\sum_{b \preceq a} \zeta_{l}(b, a) C(b)\right) \\
& =\sum_{b \preceq a} \zeta_{l}(b, a) \psi(C(b)) \\
& =\sum_{b \preceq a} \zeta_{l}(b, a)\left(\sum_{c \preceq b} \zeta_{l}^{-1}(c, b) c\right) \\
& =\sum_{c \preceq a} c \sum_{c \preceq b \preceq a} \zeta_{l}^{-1}(c, b) \zeta_{l}(b, a) \\
& =\sum_{c \preceq a} c \delta(c, a)=a
\end{aligned}
$$

and a similar argument shows that $\varphi(\psi(C(a))=C(a)$.

For future reference we state clearly the following immediate corollary of Theorem 4.2 and Lemma 4.3

Theorem 4.4. If $\unlhd_{l}$ is contained in some (principally finite) partial order then $\varphi$ is an isomorphism of $\mathbb{k}$-algebras if and only if the generalized right ample condition holds in $S$.

We will see later that $\unlhd_{l}$ is indeed contained in a partial order for a few natural cases.

Remark 4.5. The advantage of Theorem 4.4 is that it is often much easier to study the category algebra $\mathbb{k} \mathcal{C}$ and its representations than the original semigroup algebra $\mathbb{k} S$. For many natural semigroups the associated category is EI [15, 20], locally trivial [24] or a poset [16]. The representation theory of such categories is understood to a certain extent (see for instance [14, 29]). 


\section{$5 \quad$ E-Ehresmann semigroups}

Recall that a reduced $E$-Fountain semigroup is called $E$-Ehresmann if the set of idempotents $E$ is a subsemilattice (or a subband, by Lemma 3.9).

We want to show that in the case of $E$-Ehresmann semigroups the generalized and standard right ample identities are equivalent.

Proposition 5.1. Let $S$ be an E-Ehresmann semigroup. Then the right ample identity holds if and only if the generalized right ample identity holds.

Proof. In fact, this follows indirectly from Theorem 4.4 and [28, Theorem 4.4] since both properties are equivalent to $\varphi$ being an isomorphism, but we give a simple direct proof. In view of Proposition 3.14, it is enough to show that the generalized right ample identity implies the standard one. Let $a \in S$ and $e \in E$. If $E$ is a subband then for every $f \in E$ we have $e\left(a(e a f)^{*}\right)^{+} \in E$ and therefore

$$
\left(e\left(a(e a f)^{*}\right)^{+}\right)^{*}=e\left(a(e a f)^{*}\right)^{+} .
$$

The assumption that $S$ satisfies the generalized right ample identity implies

$$
e\left(a(e a f)^{*}\right)^{+}=\left(a(e a f)^{*}\right)^{+}
$$

so $e$ is left identity of $\left(a(e a f)^{*}\right)^{+}$. Since $a(e a f)^{*} \widetilde{\mathcal{R}}_{E}\left(a(e a f)^{*}\right)^{+}$this is equivalent to

$$
e a(e a f)^{*}=a(e a f)^{*}
$$

Now we can substitute $f=a^{*}$ and obtain

$$
e a=e a(e a)^{*}=e a\left(e a a^{*}\right)^{*}=a\left(e a a^{*}\right)^{*}=a(e a)^{*}
$$

as required.

We have already seen (Corollary 3.13) that if $S$ is an E-Ehresmann semigroup then $\leq_{l}=\unlhd_{l}$ so $\unlhd_{l}$ is a partial order. In this case Theorem 4.4 is already known. The fact that $\varphi$ is an isomorphism of $\mathbb{k}$-algebras was proved by the author in $[21,22$. The necessity of the right ample property was proved by Wang in [28, Lemma 4.3]. In fact, Wang proved a version of Theorem 4.4 for the class of right $P$-restriction locally Ehresmann $P$-Ehresmann semigroups (for definitions of these notions, see [11, 28]). We leave open the problem of finding a unified generalization for Theorem 4.2 and Wang's result [28, Theorem 4.4]. 


\section{Catalan monoid}

A function $f:[n] \rightarrow[n]$ (where $[n]=\{1, \ldots, n\}$ ) is called order-preserving if $i \leq j \Longrightarrow f(i) \leq f(j)$ for every $i, j \in[n]$ and order-increasing if $i \leq f(i)$ for every $i \in[n]$. Denote by $\mathrm{C}_{n}$ the monoid of all order-preserving and order-increasing functions $f:[n] \rightarrow[n]$, called the Catalan monoid. The name comes from the fact that its size is the $n$-th Catalan number [5, Theorem 14.2.8]. The Catalan monoid is $\mathcal{J}$-trivial [26, Proposition 17.17] and it is well known that every $\mathcal{J}$-trivial monoid is $E$-Fountain for $E=E(S)$ [17, Corollary 3.2]. It is also known that in a $\mathcal{J}$-trivial monoid $e f=e \Longleftrightarrow f e=e \Longleftrightarrow e \leq \mathcal{J} f$ for $e, f \in E(S)$ ([2, Lemma 3.6]) so every $\mathcal{J}$-trivial monoid is in fact a reduced $E$-Fountain monoid. We define a partial order $\preceq_{n}$ on subsets of $[n]$ in the following way. For two subsets $X=\left\{x_{1}<\ldots<x_{k}\right\}$ and $Y=\left\{y_{1}<\ldots<y_{r}\right\}$ we define $X \preceq_{n} Y$ if $k=r$ (i.e., $|X|=|Y|$ ) and $x_{i} \leq y_{i}$ for every $1 \leq i \leq k$. It is well known (see [16]) that elements of $\mathrm{C}_{n+1}$ are in one-to-one correspondence with pairs $(X, Y)$ where $X, Y \subseteq[n]$ and $X \preceq_{n} Y$. In other words, there is a one-to-one correspondence between elements of $\mathrm{C}_{n+1}$ and elements of $\preceq_{n}$ viewed as a set of ordered pairs. Explicitly, given such a pair $(X, Y)$ we define

$$
f_{X, Y}(i)= \begin{cases}y_{1} & \text { if } 1 \leq i \leq x_{1} \\ y_{j} & \text { if } x_{j-1}<i \leq x_{j} \\ n+1 & \text { if } x_{k}<i\end{cases}
$$

It is easy to verify that $f_{X, Y} \in \mathrm{C}_{n+1}$. Note that $\operatorname{im}\left(f_{X, Y}\right) \backslash\{n+1\}=Y$ and that

$$
\operatorname{ker}\left(f_{X_{1}, Y_{1}}\right)=\operatorname{ker}\left(f_{X_{2}, Y_{2}}\right) \Longleftrightarrow X_{1}=X_{2}
$$

(where $\operatorname{im}(f)$ is the image of $f$ and $\operatorname{ker}(f)$ is the equivalence relation on $[n]$ defined by $\left(x_{1}, x_{2}\right) \in \operatorname{ker} f$ if and only if $\left.f\left(x_{1}\right)=f\left(x_{2}\right)\right)$. Note also that $x_{i}$ is the maximal element whose image is $y_{i}$. Recall that $f:[n] \rightarrow[n]$ is an idempotent if and only if $f(i)=i$ for every $i \in \operatorname{im}(f)$. Therefore $f_{X, Y}$ is an idempotent if and only if $X=Y$. In particular, there is a one-to-one correspondence between idempotents in $\mathrm{C}_{n+1}$ and subsets of $[n]$. For every $Z \subseteq[n]$ we denote by $e_{Z}$ the idempotent corresponding to $Z$. Explicitly (see [26, Proposition 17.18]):

$$
e_{Z}(i)=\min \{z \in Z \cup\{n+1\} \mid i \leq z\}
$$

The following proposition is part of [26, Proposition 17.20] (with notation $f^{-}$instead of our $f^{*}$ ).

Proposition 6.1. Let $f_{X, Y} \in \mathrm{C}_{n+1}$ then $\left(f_{X, Y}\right)^{*}=e_{X}$ and $\left(f_{X, Y}\right)^{+}=e_{Y}$.

As an immediate corollary we have: 
Corollary 6.2. Let $f_{X_{1}, Y_{1}}, f_{X_{2}, Y_{2}} \in \mathrm{C}_{n+1}$ then

$$
\begin{aligned}
f_{X_{1}, Y_{1}} \widetilde{\mathcal{L}}_{E} f_{X_{2}, Y_{2}} \Longleftrightarrow X_{1}=X_{2} \Longleftrightarrow \operatorname{ker}\left(f_{X_{1}, Y_{1}}\right)=\operatorname{ker}\left(f_{X_{2}, Y_{2}}\right) \\
f_{X_{1}, Y_{1}} \widetilde{\mathcal{R}}_{E} f_{X_{2}, Y_{2}} \Longleftrightarrow Y_{1}=Y_{2} \Longleftrightarrow \operatorname{im}\left(f_{X_{1}, Y_{1}}\right)=\operatorname{im}\left(f_{X_{2}, Y_{2}}\right) .
\end{aligned}
$$

Lemma 6.3. The Catalan monoid satisfies the congruence condition.

Proof. Take two functions $f, g \in \mathrm{C}_{n+1}$. For $\widetilde{\mathcal{L}}_{E}$ to be a right congruence we need to show that $(f g)^{*}=\left(f^{*} g\right)^{*}$ or equivalently, $\operatorname{ker}(f g)=\operatorname{ker}\left(f^{*} g\right)$. Indeed,

$$
\begin{aligned}
\left(a_{1}, a_{2}\right) & \in \operatorname{ker}(f g) \Longleftrightarrow f g\left(a_{1}\right)=f g\left(a_{2}\right) \Longleftrightarrow\left(g\left(a_{1}\right), g\left(a_{2}\right)\right) \in \operatorname{ker} f \\
& \Longleftrightarrow\left(g\left(a_{1}\right), g\left(a_{2}\right)\right) \in \operatorname{ker} f^{*} \Longleftrightarrow f^{*} g\left(a_{1}\right)=f^{*} g\left(a_{2}\right) \\
& \Longleftrightarrow\left(a_{1}, a_{2}\right) \in \operatorname{ker}\left(f^{*} g\right) .
\end{aligned}
$$

To show that $\widetilde{\mathcal{R}}_{E}$ is a left congruence we need to show that $(f g)^{+}=\left(f g^{+}\right)^{+}$or equivalently, $\operatorname{im}(f g)=\operatorname{im}\left(f g^{+}\right)$. Indeed,

$$
\begin{aligned}
b \in \operatorname{im}(f g) & \Longleftrightarrow \exists a \in \operatorname{im}(g), \quad f(a)=b \Longleftrightarrow \exists a \in \operatorname{im}\left(g^{+}\right), \quad f(a)=b \\
& \Longleftrightarrow b \in \operatorname{im}\left(f g^{+}\right) .
\end{aligned}
$$

Note that the objects of the associated category are subsets of $[n]$ and if $X, Y \subseteq[n]$ then there is a unique morphism from $X$ to $Y$ if and only if $X \preceq_{n} Y$. Therefore the associated category is just the poset $\preceq_{n}$ viewed as a category.

Our next goal is to prove that the generalized right ample identity holds in the Catalan monoid. The proof given here is essentially taken from [16], but we reformulate it in the language of $E$-Fountain monoids. We first need the following definition.

Definition 6.4. Let $f \in C_{n+1}$ and let $Z \subseteq[n]$, we say that $Z$ is a partial cross section (PCS) of $f$ if $n+1 \notin f(Z)$ and $\left.f\right|_{Z}$ is injective (where $\left.f\right|_{Z}$ is the restriction of $f$ to the set $Z$ ).

Proposition 6.5. Let $f \in C_{n+1}$ and let $Z \subseteq[n]$ then $\left(f e_{Z}\right)^{*}=e_{Z}$ if and only if $Z$ is a PCS of $f$.

Proof. First assume $\left(f e_{Z}\right)^{*}=e_{Z}$. Let $z \in Z$. Since $e_{Z}$ and $f e_{Z}$ have the same kernel and $e_{Z}(z)=z \neq n+1=e_{Z}(n+1)$ then $f(z)=f e_{Z}(z) \neq f e_{Z}(n+1)=n+1$. Now if $f(z)=f\left(z^{\prime}\right)$ for some $z, z^{\prime} \in Z$ then $f e_{Z}(z)=f e_{Z}\left(z^{\prime}\right)$ hence $\left(z, z^{\prime}\right) \in \operatorname{ker}\left(f e_{Z}\right)$. Therefore, $\left(z, z^{\prime}\right) \in \operatorname{ker}\left(e_{Z}\right)$ so $z=e_{Z}(z)=e_{Z}\left(z^{\prime}\right)=z^{\prime}$ and $\left.f\right|_{Z}$ is indeed injective. In the other direction assume $Z$ is a PCS of 
$f$. We need to prove that $\operatorname{ker}\left(f e_{Z}\right)=\operatorname{ker}\left(e_{Z}\right)$. It is clear that $\operatorname{ker}\left(e_{Z}\right) \subseteq \operatorname{ker}\left(f e_{Z}\right)$. For the other containment assume $\left(x, x^{\prime}\right) \in \operatorname{ker}\left(f e_{Z}\right)$ so $f e_{Z}(x)=f e_{Z}\left(x^{\prime}\right)$. First note that

$$
f e_{Z}(x)=f e_{Z}\left(x^{\prime}\right)=n+1 \Longleftrightarrow e_{Z}(x)=e_{Z}\left(x^{\prime}\right)=n+1
$$

because $\operatorname{im}\left(e_{Z}\right)=Z \cup\{n+1\}$ and $n+1 \notin f(Z)$. The other option is that $e_{Z}(x), e_{Z}\left(x^{\prime}\right) \in Z$. In this case the fact that $\left.f\right|_{Z}$ is injective implies that

$$
f e_{Z}(x)=f e_{Z}\left(x^{\prime}\right) \Longrightarrow e_{Z}(x)=e_{Z}\left(x^{\prime}\right)
$$

so $\operatorname{ker}\left(f e_{Z}\right) \subseteq \operatorname{ker}\left(e_{Z}\right)$ as required.

Corollary 6.6. If $h \unlhd_{l} f$ for $h, f \in \mathrm{C}_{n+1}$ and $h^{*}=e_{Z}$ where $Z \subseteq[n]$ then $Z$ is a PCS of $f$.

Proof. We have $h=f e_{Z}$ so $e_{Z}=h^{*}=\left(f e_{Z}\right)^{*}$ and the result follows by Proposition 6.5

Theorem 6.7. The Catalan monoid $\mathrm{C}_{n+1}$ satisfies the generalized right ample condition.

Proof. We use the equivalent condition 3 given in Lemma4.1. Assume $h \unlhd_{l} f g$ for some $f, g, h \in \mathrm{C}_{n+1}$ and denote $h^{*}=e_{Z}$. Since $h \unlhd_{l} f g$ we know that $Z$ is a PCS of $f g$ by Corollary [6.6, Denote $W=\operatorname{im}\left(g e_{Z}\right) \backslash\{n+1\}$ and note that for every $w \in W$ there exists $z \in Z$ such that $g(z)=g e_{Z}(z)=w$. We need to prove that

$$
\left(f\left(g e_{Z}\right)^{+}\right)^{*}=\left(g e_{Z}\right)^{+} .
$$

According to Proposition 6.5 and Proposition 6.1 we need to show that $W$ is a PCS of $f$. Take $w \in W$ and let $z \in Z$ such that $g(z)=w$, then $f(w)=f g(z) \neq n+1$ since $Z$ is a PCS of $f g$. Now take $w, w^{\prime} \in W$ such that $f(w)=f\left(w^{\prime}\right)$. Take $z, z^{\prime} \in Z$ such that $g(z)=w$ and $g\left(z^{\prime}\right)=w^{\prime}$. Then $f g(z)=f g\left(z^{\prime}\right)$ so $z=z^{\prime}$ since $\left.f g\right|_{Z}$ is injective.

Since its idempotents do not form a subband (for $n \geq 3$ ), the Catalan monoid $\mathrm{C}_{n}$ is another example of a semigroup which satisfies the generalized right ample condition but not the standard one. A concrete simple counter-example is given in the following example:

Example 6.8. Consider the idempotents $e_{1}, e_{2} \in \mathrm{C}_{3}$ defined by

$$
e_{1}(i)=\left\{\begin{array}{ll}
2 & i=1,2 \\
3 & i=3
\end{array}, \quad e_{2}(i)= \begin{cases}1 & i=1 \\
3 & i=2,3\end{cases}\right.
$$

so

$$
e_{1} e_{2}(i)= \begin{cases}2 & i=1 \\ 3 & i=2,3\end{cases}
$$


It is easy to see that $\left(e_{1} e_{2}\right)^{*}=e_{2}$ as $\operatorname{ker} e_{2}=\operatorname{ker} e_{1} e_{2}$. Therefore,

$$
e_{2}\left(e_{1} e_{2}\right)^{*}=e_{2} e_{2}=e_{2} \neq e_{1} e_{2}
$$

so the right ample condition does not hold.

Remark 6.9. Example 6.8 also shows that the relation $\unlhd_{l}$ in $\mathrm{C}_{n}$ is not transitive and hence not even a preorder. It is clear that $e_{1} \unlhd_{l}$ id (where id is the identity function) and $e_{1} e_{2} \unlhd_{l} e_{1}$. However, $e_{1} e_{2}$ is not an idempotent hence $e_{1} e_{2} \sharp_{l}$ id.

Fortunately, it is easy to see that $\unlhd_{l}$ is contained in a partial order.

Lemma 6.10. Let $S$ be a reduced E-Fountain semigroup. If $S$ is $\mathcal{R}$-trivial then $\unlhd_{l}$ is contained in a partial order.

Proof. If $a \unlhd_{l} b$ then $a=b a^{*}$ so $a \leq_{\mathcal{R}} b$ hence $\unlhd_{l} \subseteq \leq_{\mathcal{R}}$. The relation $\leq_{\mathcal{R}}$ is a partial order if and only if $S$ is $\mathcal{R}$-trivial.

In particular, $\leq_{\mathcal{R}}$ is a partial order in the Catalan monoid which is a $\mathcal{J}$-trivial monoid. In conclusion, the results in this section and Theorem 4.4 imply the following corollary:

Theorem 6.11. Let $\mathbb{k}$ be a unital commutative ring. There is an isomorphism of algebras $\mathbb{k}^{\mathrm{C}_{n+1}} \simeq \mathbb{k}\left[\preceq_{n}\right]$. In particular, $\mathbb{k}_{n+1}$ is an incidence algebra.

Remark 6.12. This isomorphism is precisely the one given in [16, Theorem 3.1].

As a final observation, we consider also the generalized left ample condition.

Proposition 6.13. The Catalan monoid $\mathrm{C}_{n+1}$ satisfies the generalized left ample condition.

Proof. Note that $\left(e_{Z} g\right)^{+}=e_{Z}$ means that $e_{Z} g$ and $e_{Z}$ have the same image. In other words, for every $z \in Z$ there exists $x$ such that $e_{Z} g(x)=z$. In this case we say that $g$ is a multi cross section (MCS) of $Z$. We use the dual of condition 3 given in Lemma 4.1

$$
h \unlhd_{r} f g \Longrightarrow\left(\left(h^{+} f\right)^{*} g\right)^{+}=\left(h^{+} f\right)^{*}
$$

If $h \unlhd_{r} f g$ and $h^{+}=e_{Z}$ then $e_{Z}=h^{+}=\left(h^{+} f g\right)^{+}=\left(e_{Z} f g\right)^{+}$so $f g$ is an MCS of $Z$. Denote $e_{W}=\left(e_{Z} f\right)^{*}$. We need to prove that $g$ is an MCS of $W$. Let $w \in W$, we need to find $x$ such that $e_{W} g(x)=w=e_{W}(w)$. Note that $e_{W}$ and $e_{Z} f$ have the same kernel so this is equivalent to finding $x$ such that $e_{Z} f g(x)=e_{Z} f(w)$. Set $z=e_{Z} f(w)$ so $z \in \operatorname{im}\left(e_{Z}\right)$. If $z=n+1$ then $e_{Z} f(w)=e_{Z} f(n+1)$ so $w=e_{W}(w)=e_{W}(n+1)=n+1$, a contradiction. Therefore, $z \in \operatorname{im}\left(e_{Z}\right) \backslash\{n+1\}=Z$. Since $f g$ is an MCS of $Z$ there exists an $x$ such that $e_{Z} f g(x)=z$ as required. 


\section{References}

[1] Sterling K Berberian. Baer rings and baer *-rings. The University of Texas at Austin, Registered US Copyright Office March, 1988.

[2] Tom Denton, Florent Hivert, Anne Schilling, and Nicolas M. Thiéry. On the representation theory of finite j-trivial monoids. Sém. Lothar. Combin., 64:Art. B64d, 44, 2010/11.

[3] James East and Robert D. Gray. Ehresmann theory and partition monoids. J. Algebra, 579:318-352, 2021.

[4] David J. Foulis. Baer *-semigroups. Proc. Amer. Math. Soc., 11:648-654, 1960.

[5] Olexandr Ganyushkin and Volodymyr Mazorchuk. Classical finite transformation semigroups, volume 9 of Algebra and Applications. Springer-Verlag London Ltd., London, 2009. An introduction.

[6] Victoria Gould. Notes on restriction semigroups and related structures; formerly (weakly) left E-ample semigroups, 2010.

[7] Victoria Gould. Restriction and Ehresmann semigroups. In Proceedings of the International Conference on Algebra 2010, pages 265-288. World Sci. Publ., Hackensack, NJ, 2012.

[8] Xiaojiang Guo and Lin Chen. Semigroup algebras of finite ample semigroups. Proc. Roy. Soc. Edinburgh Sect. A, 142(2):371-389, 2012.

[9] Florent Hivert and Nicolas M. Thiéry. The Hecke group algebra of a Coxeter group and its representation theory. J. Algebra, 321(8):2230-2258, 2009.

[10] John M. Howie. Fundamentals of semigroup theory, volume 12 of London Mathematical Society Monographs. New Series. The Clarendon Press Oxford University Press, New York, 1995. Oxford Science Publications.

[11] Peter R. Jones. A common framework for restriction semigroups and regular $*$-semigroups. $J$. Pure Appl. Algebra, 216(3):618-632, 2012.

[12] M. V. Lawson. Semigroups and ordered categories. I. The reduced case. J. Algebra, 141(2):422$462,1991$.

[13] Mark V. Lawson. Rees matrix semigroups. Proc. Edinburgh Math. Soc. (2), 33(1):23-37, 1990.

[14] Liping Li. A characterization of finite EI categories with hereditary category algebras. $J$. Algebra, 345:213-241, 2011. 
[15] Stuart Margolis and Itamar Stein. Ehresmann semigroups whose categories are EI and their representation theory. J. Algebra, 585:176-206, 2021.

[16] Stuart Margolis and Benjamin Steinberg. The algebra of the catalan monoid as an incidence algebra: A simple proof. arXiv preprint arXiv:1806.06531, 2018.

[17] Stuart Margolis and Benjamin Steinberg. Projective indecomposable modules and quivers for monoid algebras. Comm. Algebra, 46(12):5116-5135, 2018.

[18] Louis Solomon. The Burnside algebra of a finite group. J. Combinatorial Theory, 2:603-615, 1967.

[19] Richard P. Stanley. Enumerative combinatorics. Vol. 1, volume 49 of Cambridge Studies in Advanced Mathematics. Cambridge University Press, Cambridge, 1997. With a foreword by Gian-Carlo Rota, Corrected reprint of the 1986 original.

[20] Itamar Stein. The representation theory of the monoid of all partial functions on a set and related monoids as EI-category algebras. J. Algebra, 450:549-569, 2016.

[21] Itamar Stein. Algebras of Ehresmann semigroups and categories. Semigroup Forum, 95(3):509$526,2017$.

[22] Itamar Stein. Erratum to: Algebras of Ehresmann semigroups and categories. Semigroup Forum, 96(3):603-607, 2018.

[23] Itamar Stein. The global dimension of the algebra of the monoid of all partial functions on an $n$-set as the algebra of the EI-category of epimorphisms between subsets. J. Pure Appl. Algebra, 223(8):3515-3536, 2019.

[24] Itamar Stein. Representation theory of order-related monoids of partial functions as locally trivial category algebras. Algebr. Represent. Theory, 23(4):1543-1567, 2020.

[25] Benjamin Steinberg. Möbius functions and semigroup representation theory. J. Combin. Theory Ser. A, 113(5):866-881, 2006.

[26] Benjamin Steinberg. Representation theory of finite monoids. Universitext. Springer, Cham, 2016.

[27] Tim Stokes. Domain and range operations in semigroups and rings. Comm. Algebra, 43(9):3979-4007, 2015.

[28] Shoufeng Wang. On algebras of $P$-Ehresmann semigroups and their associate partial semigroups. Semigroup Forum, 95(3):569-588, 2017. 
[29] Peter Webb. An introduction to the representations and cohomology of categories. In Group representation theory, pages 149-173. EPFL Press, Lausanne, 2007. 